\title{
A REMARK ON POWER SERIES RINGS
}

\author{
P. M. COHN \\ In memory of Pere Menal
}

\begin{abstract}
A trivializability principle for local rings is described which leads to a form of weak algorithm for local semifirs with a finitely generated maximal ideal whose powers meet in zero.
\end{abstract}

1. The ring of power series in several non-commuting indeterminates over a field has been characterized by the inverse weak algorithm relative to a suitable filtration (cf. [1], [4]). As an example one may take a fir $R$ with an ideal a such that $R / \mathfrak{a}$ is a skew ficld $([4$, Cor. 2.9.16]); the filtration in this case is the a-adic filtration. This shows that any fir $R$ which is a local ring with maximal ideal $\mathrm{m}$ satisfies the inverse weak algorithm relative to the $m$-adic filtration. Of course the power series ring itself is not a fir, but we get a fir by taking the subring of all rational power series $([4$, p. 460]).

However, the proof in [4] does not extend to semifirs, or even to onesided firs. Our object here is to describe a similar result for semifirs; the only additional hypothesis needed is that the ring is local with a maximal ideal which is finitely generated, as right ideal and whose powers intersect in zero. We shall also describe a form of trivializability for local rings (in Th. 1) which does not seem to have been noticed before.

2. Throughout, all rings are associative, with unit element $1 \neq 0$ (i.e. non-trivial); undefined terms are as in [4].

We recall from $[2$, Th. 1 $\}$ that any left or right semihereditary local ring is a semifir. Such rings satisfy the following strong dependence condition:

D. Given $a_{1}, \ldots, a_{n} \in R$, if the $a_{i}$ are right linearly dependent over $R$ :

$$
\sum a_{i} b_{\imath}=0, \quad b_{i} \in R, b_{i} \text { not all } 0,
$$


then the $a_{i}$ satisfy a relation (1) in which one of the coefficients $b_{i}$ is unit.

It is clear that any non-trivial ring satisfying $D$ is a semifir. More generally, we have the following result, which describes the rings satisfying this condition:

Theorem 1. A non-trivial ring $R$ satisfies condition $D$ if and only if $R$ is a semifir and a local ring.

Proof: Assume that $R$ is non-trivial and satisfies $D$. Then in any right linearly dependent set, one element can be written as a linear combination of the rest, and an induction on the number of elements shows $R$ to be a semifir. To verify that $R$ is a local ring assume the contrary. Then there exist non-units $a, b \in R$ such that $a+b=1$. So $a, b \neq 0$ and $a b=(1-b) b=b(1-b)$, so $a, b$ are right linearly dependent and by $D$; one must be dcpendent on the other, say $b=a u$. Therefore $a(1+u)=1$, but this would mean that $a$ is a unit, a contradiction which shows $R$ to be a local ring (cf. [4, Prop. 0.5.4, p. 22]).

Conversely, assume that $R$ is a semifir and a local ring. Given a relation (1), there is an invertible matrix $P=\left(p_{i j}\right)$ trivializing (1) (cf. $[4$, Th. 1.1 .1$, p. 65]), thus

$$
\sum a_{i} p_{i j}=0 \text { for some } j
$$

Since $P$ is invertible, each column contains a unit, so for a given $j$, there exists $i$ such that $p_{i j}$ is a unit, and this proves that $D$ holds for $R$.

3. Let $R$ be a local scmihcreditary ring with maximal ideal $m$ and assume further that $\mathrm{m}$ is finitely generated as right ideal. As we have seen, $R$ is a semifir, therefore $m$ is free as right $R$-module. We shall implicitly use the following auxiliary result:

Lemma 2. Let $R$ be a ring with a right ideal a which is free of finite rank. If $x_{1}, \ldots, x_{r}$ is a basis for a as right ideal, then for any $n \geq 1$,

$$
\sum x_{i} a_{i} \in \mathrm{a}^{n+1} \Longrightarrow a_{i} \in \mathrm{a}^{n} \quad(i=1, \ldots, r)
$$

Proof: It is clear that $a^{n}$ is generated by all power products of the $x$ 's of degrec $n$, say $u_{1}, \ldots, u_{N}$, where $N=r^{n}$. Any element of $a^{n+1}$ can be written as $\sum x_{i} u_{\nu} a_{i \nu}$, where $a_{i \nu} \in R$. Hence, when (2) is given, we have $\sum x_{i} a_{i}=\sum x_{i} u_{\nu} a_{i \nu}$; since the $x$ 's are right linearly indepondent, we find that $a_{i}=\sum u_{\nu} a_{i \nu}$ and this lies in $a^{n}$, as claimed. 
Now let $R$ be a semihereditary local ring whose maximal ideal $\mathrm{m}$ is finitely generated, as right ideal. Then the m-adic filtration on $R$, given by the powers of $\mathrm{m}$ :

$$
R \supset \mathrm{m} \supset \mathrm{m}^{2} \supset \ldots,
$$

allows an order function $v$ to be defined on $R$ by

$$
v(a)=\sup \left\{n ; a \in \mathrm{m}^{n}\right\},
$$

precisely when $\cap \mathrm{m}^{n}=0$.

We have now satisfied all the hypotheses of Th. 2.2 of [3] (cf. also [4, Cor. 2.9.13]): $R / \mathrm{m}$ is a skew field, $\mathrm{m}$ is free and $\mathrm{nm}^{n}=0$. This theorem tells us that $R$ satisfies the inverse weak algorithm relative to the m-adic filtration. Moreover, the elements of $R$ have a power series representation which also applies to the elements of the m-adic completion $\hat{R}$ of $R$. We can state our result as follows:

Theorem 3. Let $R$ be a local semihereditary ring with maximal ideal $\mathrm{m}$ and assume that $\mathrm{m}$ is finitely generated as right ideal. Then $R$ satisfies the inverse weak algorithm relative to the $\mathrm{m}$-adic filtration if and only if $\cap \mathrm{m}^{n}=0$. Moreover, $R$ can be embedded in its m-adic completion $\hat{R}$.

Let $K=R / \mathrm{m}$ be the residue class field of $R$, take a set $\bar{K}$ of representatives of $K$ in $R$ (with 0 represented by itself, for simplicity), and let $x_{1}, \ldots, x_{r}$ be a right $R$-basis of $\mathrm{m}$. Then every element of the completion $\hat{R}$ con be uniquely written as a convergent series

$$
\sum x_{1} \alpha_{l}, \quad x_{I}=x_{i_{1}}, \ldots, x_{i_{n}}, \quad \alpha_{I} \in K,
$$

where $I=\left(i_{1}, \ldots, i_{n}\right)$ runs over all finite suffix-sets, and oll such expressions represent elements of $R$.

If $m$ is not finitely generated, such a representation may not be possible, for the simple reason that now $m$ need not be free. An example is given by the one-sided fir constructed in [2] (see also [4, p. 176]); there the maximal ideal is finitely generated as right ideal, but not finitely generated (and in fact not free) as left ideal.

In our local ring $R$ the m-adic filtration may be regarded as a natural filtration, but other filtrations are possible, e.g. by assigning different positive integers as orders to $x_{1}, \ldots, x_{r}$. However, it seems that (in the situation of Th. 3) every inverse filtration on $R$ leads to an inverse weak algorithm. This is in contrast to the situation in free algebras, where it is easy to find degree functions for which the weak algorithm fails, for example the free subalgebras that are not regularly embedded, cf. $[4, p$. $330]$. 


\title{
References
}

1. P. M. CoHN, Factorization in non-commutative power series rings, Proc. Camb. Phil. Soc. 58 (1962), 452-464.

2. P. M. CoHN, Hereditary local rings, Nagoya J. Math. 27(1) (1966), 223-230.

3. P. M. COHN, On a class of rings with inverse weak algorithm, Math. Zeits. 117 (1970), I-6.

4. P. M. CоHN, "Free rings and their relations," 2nd. Ed., LMS Monographs 19, Academic Press, London and New York, 1985.

\author{
Department of Mathematics \\ University College London \\ Gower Strcet \\ London WC1E 6BT \\ ENGLAND
}

Rebut el 10 de Setembre de 1991 